University of Nebraska - Lincoln

DigitalCommons@University of Nebraska - Lincoln

September 1998

\title{
Radial distribution of electron spectra from high-energy ions
}

Francis A. Cucinotta

NASA Johnson Space Center, francis.cucinotta@unlv.edu

Robert Katz

University of Nebraska-Lincoln, rkatz2@unl.edu

John W. Wilson

NASA, Langley Research Center, Hampton, VA

Follow this and additional works at: https://digitalcommons.unl.edu/physicskatz

Part of the Physics Commons

Cucinotta, Francis A.; Katz, Robert; and Wilson, John W., "Radial distribution of electron spectra from highenergy ions" (1998). Robert Katz Publications. 61.

https://digitalcommons.unl.edu/physicskatz/61

This Article is brought to you for free and open access by the Research Papers in Physics and Astronomy at DigitalCommons@University of Nebraska - Lincoln. It has been accepted for inclusion in Robert Katz Publications by an authorized administrator of DigitalCommons@University of Nebraska - Lincoln. 


\section{Radial distribution of electron spectra from high-energy ions}

Received: 11 February 1998 / Accepted in revised form: 1 September 1998

\begin{abstract}
The average track model describes the response of physical and biological systems using radial dose distribution as the key physical descriptor. We report on an extension of this model to describe the average distribution of electron spectra as a function of radial distance from an ion. We present calculations of these spectra for ions of identical linear energy transfer (LET), but dissimilar charge and velocity to evaluate the differences in electron spectra from these ions. To illustrate the usefulness of the radial electron spectra for describing effects that are not described by electron dose, we consider the evaluation of the indirect events in microdosimetric distributions for ions. We show that folding our average electron spectra model with experimentally determined frequency distributions for photons or electrons provides a good representation of radial event spectra from high-energy ions in $0.5-2 \mu \mathrm{m}$ sites.
\end{abstract}

\section{Introduction}

The response of physical detectors and biological systems to heavy particle irradiation is of interest in space radiation protection $[1,2]$ and studies of cancer therapy with proton and heavy ion beams [3]. For over 30 years, the average or amorphous track model has successfully described the response of a wide variety of physical detectors and biological systems to heavy particle irradiation using the radial dose distribution from delta-rays about the path of the

\section{F. A. Cucinotta (四)}

NASA Johnson Space Center,

Mail Code SN, Houston TX 77058, USA

Tel.: 281-483-0968, Fax: 281-483-5276,

e-mail: Fcucinot@ems.jsc.nasa.gov

R. Katz

University of Nebraska, Lincoln, NE 68558, USA

J. W. Wilson

NASA, Langley Research Center, Hampton, VA 23668, USA ion as the key physical descriptor [4-8]. In some instances, the response of physical or biological systems may have a dependence on electron energy other than the electron linear energy transfer (LET). This is especially true for electrons with energies below $5 \mathrm{keV}$ [9-11], and this aspect of heavy particle track structure has not been considered before in the average track model. Monte-Carlo track simulation codes provide such descriptions, but are burdened by large computational times when considering high-energy ions and large radial distances from the ion's path [12]. Furthermore, in most applications with high-energy nuclei including cancer therapy and space or atmospheric radiation studies, nuclear fragmentation and energy loss processes lead to a broad spectrum of ion types and velocities $[2,13,14]$. Important examples are the understanding of biological effectiveness using a spread-out Bragg peak and of the galactic cosmic rays where charge groups from hydrogen to nickel with energies from below $1 \mathrm{MeV} / \mathrm{amu}$ to above $10 \mathrm{GeV} / \mathrm{amu}$ contribute to biological risk $[1,2]$. Such considerations point to the usefulness of analytic approaches to treat track structure for high-energy particles. In this paper, we report on the extension of the average track model to describe the average radial distribution of electron energy spectra from heavy particles. We also develop an analytic model to evaluate frequency-event spectra that includes the indirect events important for high-energy ions and the radial dependence of these spectra.

\section{Model for radial electron spectrum}

The approach of the average-track model has been to consider the primary electron spectrum from ion interactions with target atoms and to fold this spectrum with average transmission properties of electrons to obtain the spatial distribution of electron dose as a function of radial distance from the ion's path. As introduced by Kobetich and Katz [5], the radial dose is given by

$D_{\delta}(t)=-\frac{1}{2 \pi t} \sum_{i} \int \mathrm{d} \Omega \int \mathrm{d} \omega \frac{\partial}{\partial t}[E(t, \omega) \eta(t, \omega)] \frac{\mathrm{d} n_{i}}{\mathrm{~d} \omega \mathrm{d} \Omega}$ 
In Eq. (1), $\omega$ is the initial electron energy, $E$ is the residual energy of an electron with energy $\omega$ after travelling distance $t$, and $\eta(t, \omega)$ is the transmission probability that an electron with starting energy $\omega$ penetrates a depth $t$. Equation (1) includes an angular distribution for the primary electrons with energy $\omega$ and solid angle $\Omega$, and the subscript ' $\delta$ ' indicates that it is the dose contribution from ionization by secondary electrons at a radial distance $t$ from the ion's path. The input functions for the evaluation of Eq. (1) are described by Cucinotta et al. [15] and include the model of Rudd [16] for electron spectra from proton collisions and electron transport properties from $[17,18]$. A phenomenological angular distribution for electron production was described in [15], where a distribution peaking at the classical ejection angle with a width adjusted to experimental data was used, but this model fails to describe any forward or backward angle peaks in the angular distribution. Previous calculations have shown that the angular distribution has important effects on the radial distribution both at large and small radial distances, and only a minor effect at intermediate values where a $1 / t^{2}$ behavior holds. The cross-sections for electron production from protons are scaled to heavy ions using effective charge. The use of an effective charge approximation that is dependent on the ejected-electron energy would be more accurate, especially for ions with energies below about $1 \mathrm{MeV} / \mathrm{u}$.

LET can be described by integrating the radial dose distribution over all radial distances up to the maximum allowable, $t_{\mathrm{M}}$, and including other contributions such as excitations and nuclear stopping, as

$$
\mathrm{LET}=2 \pi \int_{0}^{t_{\mathrm{M}}} t \mathrm{~d} t\left[D_{\delta}(t)+D_{\mathrm{exc}}(t)\right]+\text { Nucl. Stopping }
$$

The effects of binding are contained in the delta-ray term. Brandt and Ritchie [19] have considered an ansatz for the excitation term, $D_{\text {exc }}(t)$, as

$$
D_{\mathrm{exc}}(t)=C_{\mathrm{exc}}(A, Z, \beta) \frac{\exp (-t / 2 d)}{t^{2}}
$$

with $d=\beta / 2 \omega_{r}$ with $\omega_{r}=13 \mathrm{eV}$ for water. In Eq. (2), as described by Brandt and Ritchie [19], the radial extension of excitations is confined to very small distances, as characterized by the parameter $d$. The value of the parameter $C_{\text {exc }}$ is adjusted such that after numerical integration of the terms on the right-hand side of Eq. (2), the value of LET for a given ion of mass number $(A)$, charge number $(Z)$, and energy $(E)$, is reproduced. We have used the LET representations for ions described in [2] for our calculations.

In many applications, the number of electrons as well as their energy spectrum are required to describe the response of a system. To derive the average or residual energy spectrum of electrons penetrating to a radial distance $t$ we compare Eq. (1) to the dose at $t$ that would be found from folding the residual electron spectrum, $\phi(t, E)$, with the electron stopping power $S(E)$,

$$
D(t)=\int \mathrm{d} E \phi(t, E) S(E)
$$

In considering the radial electron spectrum, only the deltaray term in Eq. (2) contributes to it. Transforming from primary electron energy $\omega$ to residual electron energy $E$ within the continuous slowing down approximation leads to the form

$$
\begin{aligned}
\phi(t, E)= & \frac{1}{2 \pi t} \sum_{i} \int \mathrm{d} \Omega \frac{S(\omega)}{S(E)} \\
& \cdot\left[\eta(t, E)+\frac{E}{S(E)} \frac{\partial \eta(t, E)}{\partial t}\right] \frac{\mathrm{d} n_{i}}{\mathrm{~d} \omega \mathrm{d} \Omega}
\end{aligned}
$$

where $\omega$ is now a function of $E$ and $t$. Equation (5) shows that the electron spectrum is attenuated through two factors: first, an overall factor of $1 / t$ for all secondary electrons, and second an additional attenuation that is dependent on the electron starting energy and depth of penetration. Equation (5) does not describe the angular dependence of electron transmission, but inclusion of an angular-dependent transmission function [20, 21] along with the angular dependence of the primary spectrum will allow the present model to be extended in this manner.

Comparisons of the radial distribution in dose for ${ }^{1} \mathrm{H}$ at $1 \mathrm{MeV}(\mathrm{LET}=27 \mathrm{keV} / \mu \mathrm{m})$ to the measurements of Wingate and Baum [22] are shown in Fig. 1a. The model agrees well with the measurement for distances of less than $10 \mathrm{~nm}$
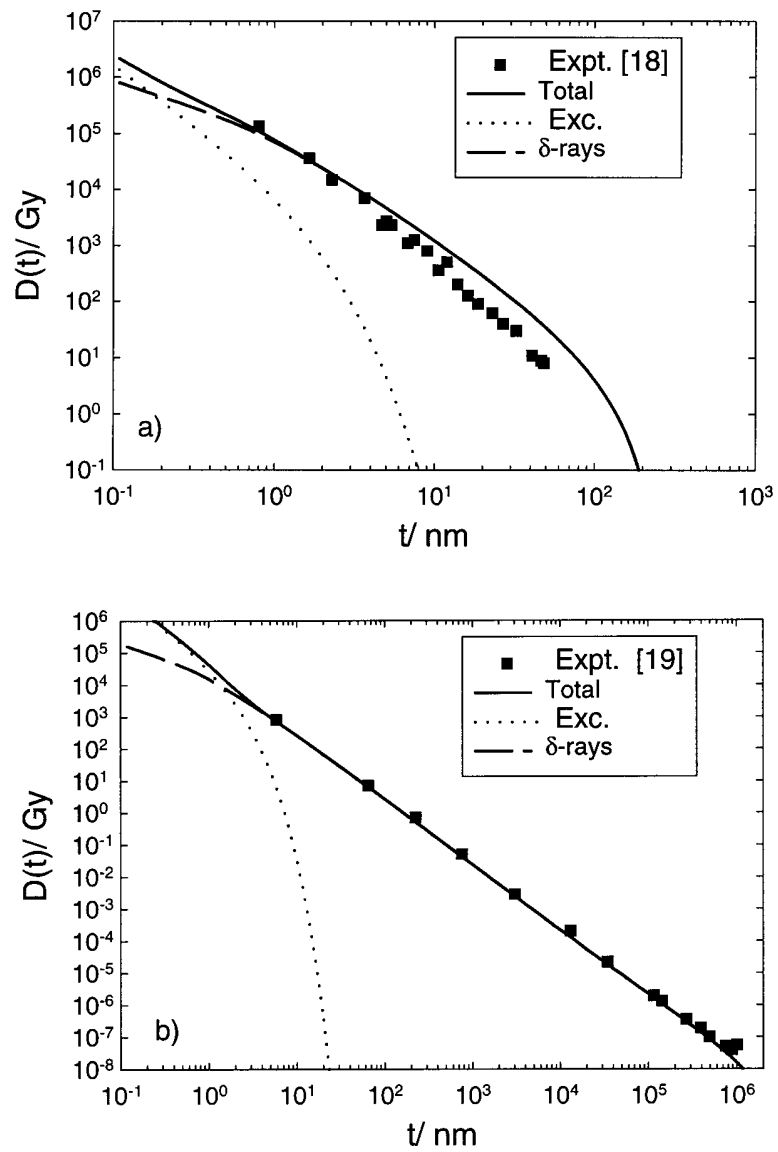

Fig. 1 Calculations of radial dose distributions and comparison to experiments $[22,26]: \mathbf{a}^{1} \mathrm{H}$ at $1 \mathrm{MeV}(\mathrm{LET}=27 \mathrm{keV} / \mu \mathrm{m}), \mathbf{b}^{20} \mathrm{Ne}$ at $377 \mathrm{MeV} / \mathrm{u}(\mathrm{LET}=31 \mathrm{keV} / \mu \mathrm{m})$ 


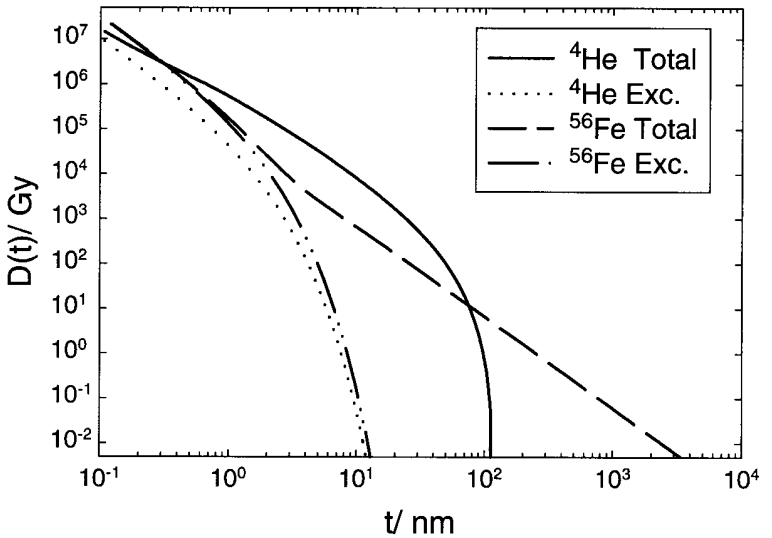

Fig. 2 Calculations of radial dose distributions for ions of $151 \mathrm{keV} /$ $\mu \mathrm{m}:{ }^{4} \mathrm{He}$ at $0.55 \mathrm{MeV} / \mathrm{u}$ and ${ }^{56} \mathrm{Fe}$ at $1 \mathrm{GeV} / \mathrm{u}$

and predicts higher values than the data at larger distances $(>10 \mathrm{~nm})$. Several comparisons of Monte-Carlo calculations [22-25] to the data of [22] have been made. These comparisons also have tended to overestimate the measurements of Wingate and Baum [22] for radial distances beyond $10 \mathrm{~nm}$ or suffered from decreased resolution due to the need for performing a large number of trials when the event frequency is low. In Fig. 1b, we compare the model to measurements [26] for ${ }^{20} \mathrm{Ne}$ at $377 \mathrm{MeV} / \mathrm{u}$, which has a LET of $31 \mathrm{keV} / \mu \mathrm{m}$, which is close to that of $1-\mathrm{MeV}$ protons. The model and measurements are in good agreement. Comparing Fig. 1a and b provides an indication of the differences in track structure that occur for ions of the same LET, but dissimilar charge and velocity. Figure 2 shows comparisons of the radial dose distribution for ions with an identical LET of $151 \mathrm{keV} / \mu \mathrm{m}:{ }^{4} \mathrm{He}$ at $0.55 \mathrm{MeV} / \mathrm{u}$ and ${ }^{56} \mathrm{Fe}$ at $1 \mathrm{GeV} / \mathrm{u}$. The above comparisons indicate differences in track structure for ions of identical LET due to track density and track width. The comparisons described next indicate further differences due to electron energy spectra.

In Figs. 3 and 4, we show calculations of the radial electron spectrum at several impact parameters. Figure 3a displays results for ${ }^{1} \mathrm{H}$ ions at $1 \mathrm{MeV}$ and Fig. $3 \mathrm{~b}$, for ${ }^{20} \mathrm{Ne}$ ions at $377 \mathrm{MeV} / \mathrm{u}$. Both of these ions have LET values close to $30 \mathrm{keV} / \mu \mathrm{m}$. Similarly, in Fig. 4, we show results for $151 \mathrm{keV} / \mu \mathrm{m}$ ions: ${ }^{4} \mathrm{He}$ ions at $0.55 \mathrm{MeV} / \mathrm{u}$ and ${ }^{56} \mathrm{Fe}$ ions at $1000 \mathrm{MeV} / \mathrm{u}$. We have plotted the spectrum from Eq. (5) as $2 \pi t E \phi(t, E)$ vs energy to show the $1 / t$ attenuation of the spectrum and since ions at larger impact parameters reach a larger number of potential target molecules (as described by the differential cross-sectional area $2 \pi t \mathrm{~d} t$ ). The results in Figs. 3 and 4 illustrate that when comparing ions of similar LET values, lower charge ions have both a confined trackwidth and a larger contribution from electrons of lower energy in comparison to a highercharged ion. Experiments with soft $x$-rays indicate increased biological effectiveness for electrons with energies less than several keV [9]. For ions of a given LET value, low charge and energy ions (LZE) may have in-
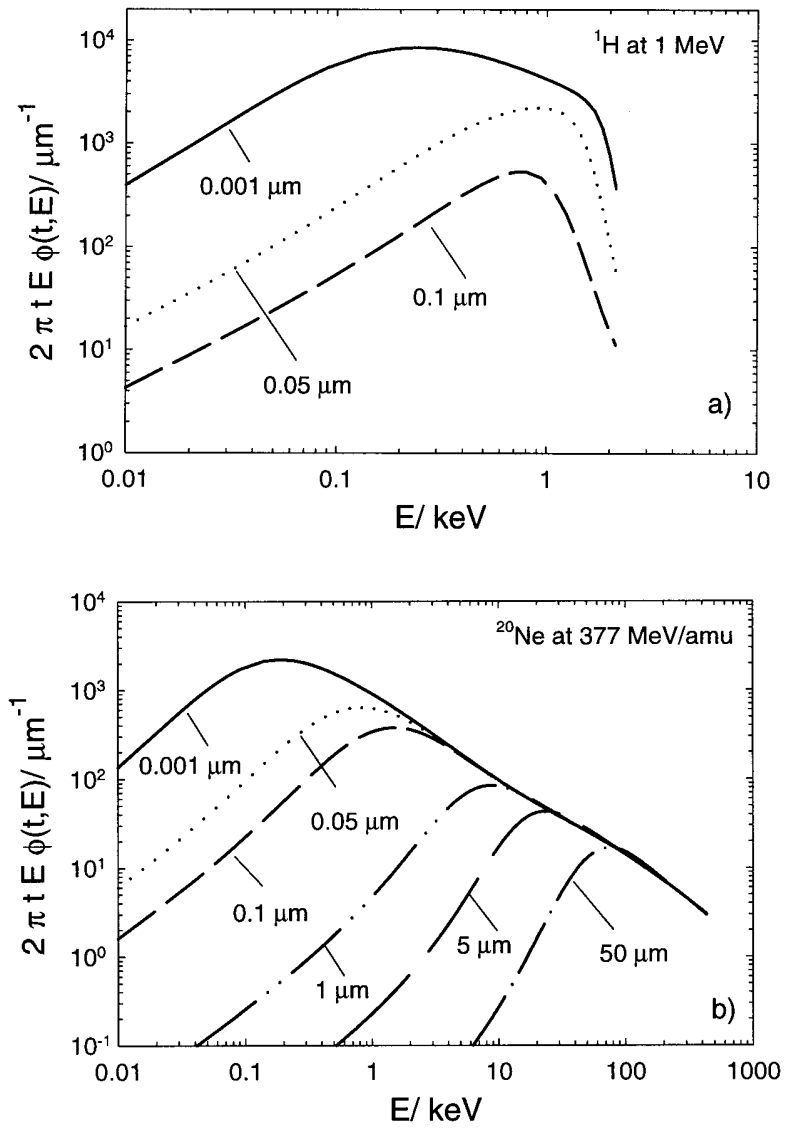

Fig. 3 Calculations of $2 \pi t E \phi(t, E)$ vs electron energy at various radial distances from ions of LET near $30 \mathrm{keV} / \mu \mathrm{m}$ : $\mathbf{a}^{1} \mathrm{H}$ at $1 \mathrm{MeV}$ $(\mathrm{LET}=27 \mathrm{keV} / \mu \mathrm{m}), \mathbf{b}^{20} \mathrm{Ne}$ at $377 \mathrm{MeV} / \mathrm{u}(\mathrm{LET}=31 \mathrm{keV} / \mu \mathrm{m})$

creased effectiveness because of the predominance of lowenergy electrons in comparison with high charge and energy ions (HZE). The secondary electron spectrum for the LZE ions is softer, both at small radial distances and at the maximal radial distances where the highest energy ejected electrons are stopped (electron track-ends). However, for large target volumes including a response dependent on alterations in spatially distributed target molecules, the present energy deposition model $[6,7]$ predicts that the effects of track width increase the effectiveness of HZE ions over LZE ions.

In Fig. 5 we show the frequency-averaged (averaged over the electron energy spectrum) and the dose-averaged (averaged over electron spectrum folded with LET) electron energies as a function of radial distance from the ion path for ions of energy 1,10,100, and $1000 \mathrm{MeV} / \mathrm{u}$. These results are approximately independent of ion charge due to the effective charge scaling used in the calculations. The average electron energy is seen to increase with distance from the track. In considering the variation of biological effectiveness with electron energy, of note is the large increase in effectiveness for electrons with energies below $5 \mathrm{keV}$ [9-11]. For larger volumes with diameters of $100 \mathrm{~nm}$ or more, electron track overlap may occur, perhaps reducing the importance of the effectiveness of electrons 

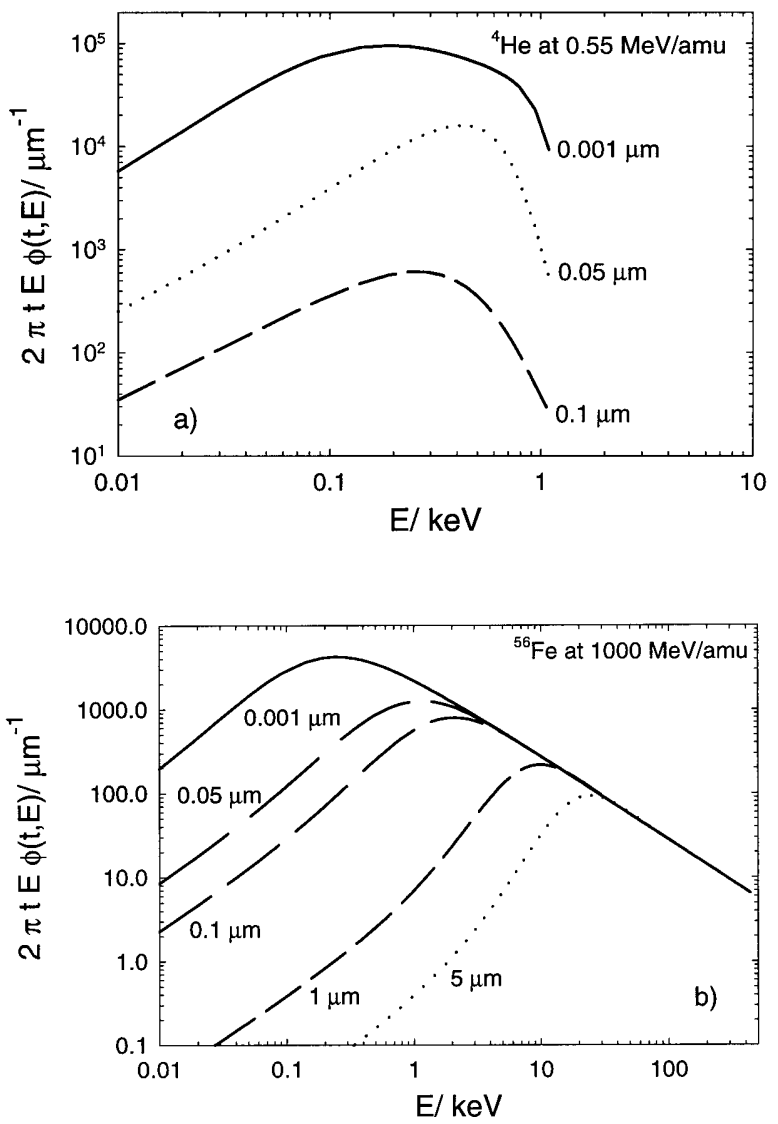

Fig. 4 Calculation of $2 \pi t E \phi(t, E)$ at various radial distances from ions of LET $=151 \mathrm{keV} / \mu \mathrm{m}: \mathbf{a}{ }^{4} \mathrm{He}$ at $0.55 \mathrm{MeV} / \mathrm{u}, \mathbf{b}^{56} \mathrm{Fe}$ at 1000 $\mathrm{MeV} / \mathrm{u}$

of varying energies. The present model offers an efficient method to model such effects. Differences in biological response have been seen between hydrogen and helium ions at the same LET [27, 28] and with low-energy heavy ions $[29,30]$, but useful comparisons of fast and slow ions have not been made. For estimating the risk to astronauts from space radiation, differences in radiation quality for ions with the same LET may have important implications. Experiments to elucidate differences would be useful for understanding radiation quality for space radiation protection where LZEs and HZEs make nearly equal contributions to LET spectra above $20 \mathrm{keV} / \mu \mathrm{m}$ [13]. The energy deposition considerations described here would suggest that these differences may be important.

\section{Application to indirect events in microdosimetry}

In the description of frequency distributions as measured by proportional counters, events arising from delta-rays produced by particles that do not pass through the sensitive volume are denoted outside or indirect events [31,32]. At high energies, a significant fraction of the LET or events arises from outside events for site sizes in the $0.5-2 \mu \mathrm{m}$
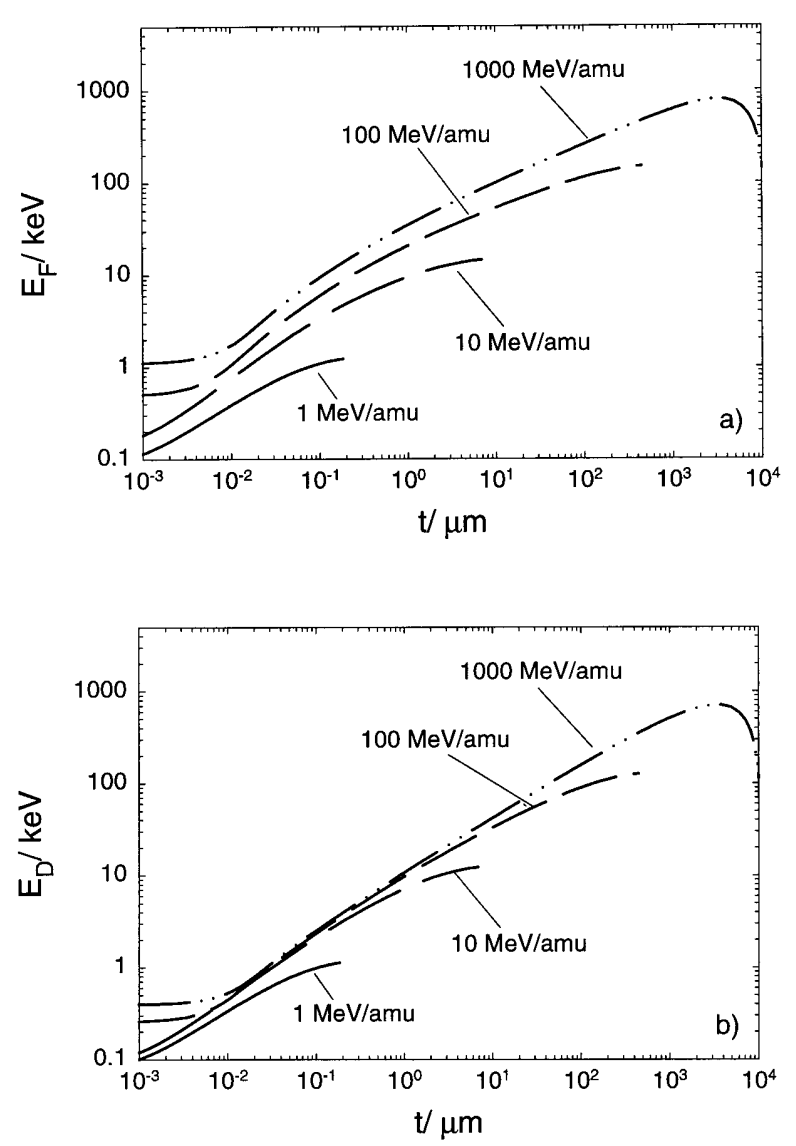

Fig. 5 Calculation of the a frequency-averaged mean electron energy and $\mathbf{b}$ dose-averaged mean electron energy as a function of radial distance from an ion's track for ions of energy 1, 10,100, and $1000 \mathrm{MeV} / \mathrm{u}$. Since we are using effective charge to scale the effects of different ions the present results are independent of ion charge

range used in most applications. For nanometer-sized sites that are expected to be important in producing DNA damage and mutations, event spectra are dominated by the outside events. Here we show that knowledge of the frequency distributions as a function of electron energy and the model of electron spectra discussed above can be used to describe the radial distribution of events from heavy particles.

The theoretical evaluation of microdosimetric spectra from electrons is difficult to treat analytically due to the small mean free path of electrons for elastic and inelastic collisions and the importance of energy and range straggling for electrons. Extensive measurements of microdosimetric spectra using photons and high-energy electrons over a large range of energies have been made with tissue equivalent proportional counters (TEPCs) [33]. For smaller site sizes, Monte-Carlo calculations of event spectra have been done for electrons with energies from 0.1 to $100 \mathrm{keV}$ $[9,10]$.

Our approach is to consider the average electron spectrum of electrons at radial distance $t$ from the ion's path and fold this distribution with results from measurements or Monte-Carlo simulations for electrons. We did not consider the angular dependence of electron transmission at 
this time. For an isotropic source of ions and a spherical volume, we consider an isotropic source of electrons incident on the sensitive volume as a reasonable first approximation. The event spectrum from indirect events is then described by

$f_{\text {out }}^{(1)}(\varepsilon, t)=\int \mathrm{d} E \phi(t, E) f_{e^{-}}(E, \varepsilon)$

where the distributions $f_{e^{-}}(E, \varepsilon)$ are the events of size $\varepsilon$ by electrons of energy $E$ in a particular volume as inferred from experiments or Monte-Carlo simulations. The lineal energy is related to the energy deposited and the mean chord-length, $c$, by $y=\varepsilon / c$ and the specific energy and the volume mass, $m$, by $z=\varepsilon / m$ where $z$ is in Gy, $y$ in $\mathrm{keV} / \mu \mathrm{m}$, and $c$ in $\mu \mathrm{m}$. For more higly charged ions, overlapping electron tracks may occur at impact parameters close to the sensitive volumes. We introduce higher order delta-ray terms using the Poisson distribution and the radial distribution of the number of events as

$$
f_{\text {out }}(\varepsilon, t)=\sum_{j=1} \frac{e^{-n(t)}}{j !} n(t)^{j} f_{\text {out }}^{(j)}(\varepsilon, t)
$$

where the $f_{\text {out }}^{(j)}$ are the convolutions of the single-event spectra and $n(t)$ is the number of events at radial distance $t$ as given by

$n(t)=\frac{D(t)}{z_{1 \mathrm{~F}}(t)}$

where $z_{1 \mathrm{~F}}(t)$ is the frequency-averaged value of the firstorder term.

Since measurements are available over a larger range of energies for photons than electrons, we have considered photon data here. We have parameterized the $y$-spectra from measurements with photons using the function

$f(y)=N\left[c a \exp (-y / a)+(1-c) \sqrt{\pi b} \exp (-y / b)^{2}\right]$

where $N$ is a normalization constant and with the parameters chosen as $b=6.5 \mathrm{keV} / \mu \mathrm{m}$. The other parameters in Eq. (9) are given as a function of photon energy as

$a=a_{1}+a_{2} \exp \left[-\left(E_{\text {photon }} / 60\right)^{1 / 2}\right]$

and

$c=1-c_{1} \exp \left(-E_{\text {photon }} / 1000\right)$

to approximate measured values for $y_{\mathrm{F}}$ and $y_{\mathrm{D}}$ as a function of photon energy. A purely exponential function leads to $y_{\mathrm{D}} / y_{\mathrm{F}}=2$, which is approximately true for photon energies below $50 \mathrm{keV}$ [33]. The second term in Eq. (9) allows for a higher ratio that occurs at higher photon energies. We compare the frequency and dose-averaged values that result from Eqs. (9-11) to measured values [33] in Fig. 6. The large scatter in experimental values precluded a statistical fit, and the parameters in Eqs. (9-11) have been fit by eye. The spectrum of Eq. (9) is fit to experiments for photon irradiation in walled counters [33]. Some differences in the response of walled and wall-less counters for photons and electrons should be expected and are not described here. In order to relate the photon energy to electron energy, we use the average secondary electron
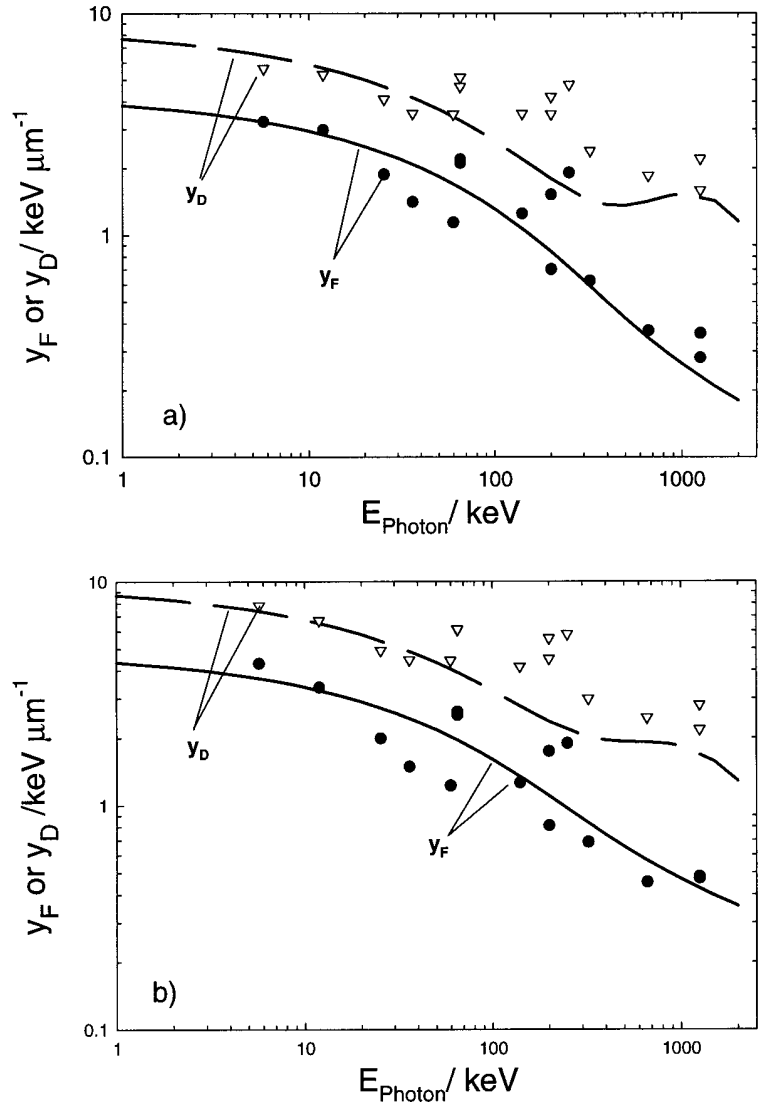

Fig. 6 Comparison of parametric model to experiments [33] for $y_{\mathrm{F}}$ and $y_{\mathrm{D}}$ vs photon energy: a $1.0 \mu \mathrm{m}$ site, $\mathbf{b} 0.5 \mu \mathrm{m}$ site

Table 1 Parameters for parametric equations of photon lineal energy distributions

\begin{tabular}{lrll}
\hline Site diameter $(\mu \mathrm{m})$ & $a_{1}$ & $a_{2}$ & $c_{1}$ \\
\hline 0.5 & 3 & 4.6 & 0.0050 \\
1.0 & 14 & 4.2 & 0.0015 \\
2.0 & 14 & 3.4 & 0.0010 \\
4.0 & 18 & 2.6 & 0.0005 \\
\hline
\end{tabular}

energy from Compton scattering and the photoelectric effect.

In Fig. 7a, we show the frequency average of the specific energy, $z_{\mathrm{F}}(t)$ as a function of radial distance from the path of $600 \mathrm{MeV} / \mathrm{u}$ Fe ion for a site of $1.3 \mu \mathrm{m}$ diameter. Values for the parameters in Eqs. (9-11) for a $1.3 \mu \mathrm{m}$ site are obtained by linear interpolation of the results in Table 1 . The model predicts higher values for the frequency average than the experiment of Metting et al. [34]. We have included terms through second-order (solid line) in Eq. (7) which provides some increase over the first-order term (dashed line) for radial distances below $3 \mu \mathrm{m}$. More accurate representations of the event spectra from electrons, the inclusion of the angular dependence of electron transmission, and the effects of charge scaling of electron components are potential areas for improving the present model. The frequency averaged values and their correlation with 

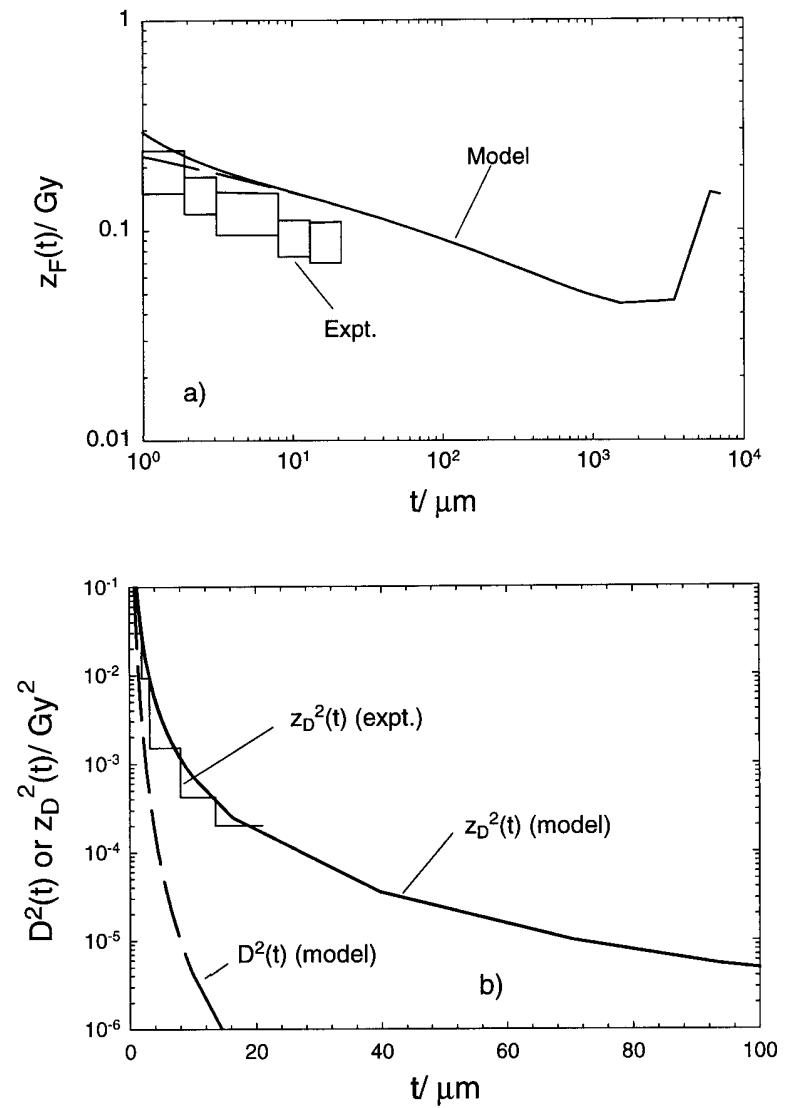

Fig. 7 Comparison of calculation to experiment [34]: a for average specific energy in $1.3 \mu \mathrm{m}$ site as a function of radial distance for 600-MeV/u Fe ions. Dashed line is first-order term in Eq. (7), and solid line is first- and second-order terms; $\mathbf{b}$ for square of the mean specific energy in $1.3 \mu \mathrm{m}$ site as a function of radial distance for 600-MeV/u Fe ions. Dashed line is first-order term in Eq. (7), and solid line is first- and second-order terms

known values for electrons and photons clearly indicate the role of delta-rays in outside events. We have plotted our results on a logarithmic scale in order to display the effects of electron track-ends that occur at the maximum radial distance. These low-energy electrons lead to an increase in the frequency average, but very few events will occur at these distances. For low-energy ions, a larger number of events will occur at the maximum radial distance of the electrons, but the electron spectrum is softer, and the relative contribution change over the width of the particles track is not as pronounced as that occurring for relativistic ions. In Fig. 7b, we show comparisons of calculation to experiment for the average value of the square of the specific energy which is given in terms of the dose-average specific energy, $z_{\mathrm{D}}(t)$, and the radial dose as

$z_{\mathrm{D}}^{2}(t)=z_{\mathrm{D}}(t) D(t)+D^{2}(t)$

Figure $7 \mathrm{~b}$ shows good agreement between experiment [34] and model. The effects of the second-order term are less pronounced than in Fig. 7a because the second term on the right-hand side of Eq. (12) dominates at small values of $t$. In Fig. 8, we show comparisons of model to experiment

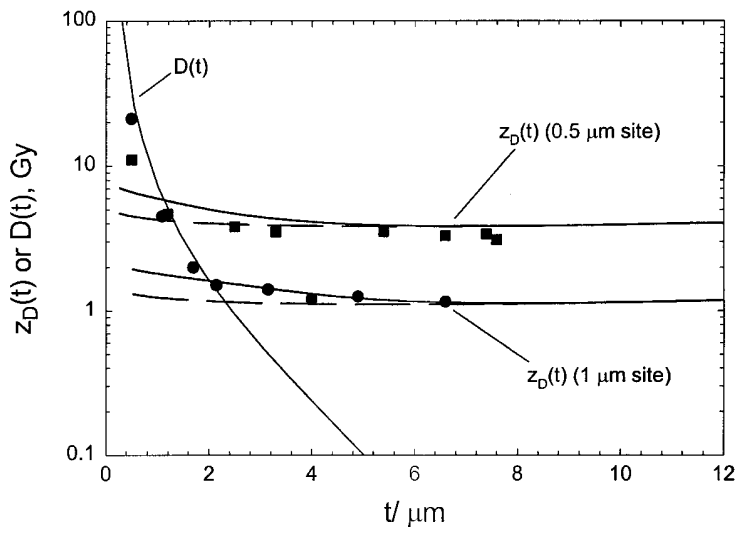

Fig. 8 Comparison of calculation to experiment [35] for dose-average specific energy in 0.5 and $1.0 \mu \mathrm{m}$ site as a function of radial distance for 14-MeV/u Ge ions. Dashed line is first-order term in Eq. (7) and solid line is first- and second-order terms

[35] for $z_{\mathrm{D}}(t)$ for $14-\mathrm{MeV} / \mathrm{u}$ Ge ions in 0.5 and $1.0 \mu \mathrm{m}$ sites. Comparing the results of Figs. 7 and 8 provides an indication of the softer electron spectrum for lower energy ions. Also, the second-order term provides a larger contribution for the higher charge Ge ion compared with Fe. The model discussed herein can be combined with treatments of direct events using path-length distributions corrected for ion straggling to provide accurate descriptions of the total event spectra from ions.

\section{Conclusions}

The approach of the average track model has been to use parametric models of electron energy deposition and the primary electron spectrum released by heavy particles to describe the effects of energy deposition of ions. The resulting spatial distribution of energy deposition can be folded with a physical or biological system's characteristic response to electrons or photons to describe the equivalent effect by ions. In this report, we have added a new aspect to this approach by considering the radial distribution of electron spectra about the ion's path. Our calculations for ions of identical LET show that along with previously reported effects of ion trackwidth, differences in the spectrum of electron energies may result in distinct effects for such ions. For a response dependent on electron energy and fluence rather than electron dose, our model allows for an efficient method to model the effects of ions. We have shown that this approach provides a method for evaluating the radial distribution of frequency-event spectra using measured results for photons (or electrons). These distributions are often neglected and are time-consuming to consider in Monte Carlo codes, especially for high-energy ions. Our method offers an accurate approach to this problem. The comparisons of the radial distribution for electron energies or specific energy may provide new insights into the success of the average-track model in de- 
scribing relative biological effectiveness for diverse radiation fields and approaches to improve this model.

Acknowledgement One of us (F. C.) would like to acknowledge useful discussions related to this work with D. T. Goodhead.

\section{References}

1. Cucinotta FA, Wilson JW, Katz R, Atwell WJ, Badhwar GD, Shavers MR (1996) Track structure and radiation transport model for space radiobiology studies. Adv Space Res 18: 183-194

2. Wilson JW, Townsend LW, Schimmerling W, Nealey JE, Khandelwal GS, Khan F, Cucinotta FA, Simonsen LS, Shinn JL, Norbury JW (1991) Transport methods and interactions for space radiation. (NASA RP 1257) National Aeronautical Space Agency, Washington D.C.

3. Blakeley EA, Tobias CA, Yang TCH, Smith KC, Lyman JT (1979) Inactivation of human kidney cells by high-energy monoenergetic heavy-ion beams. Radiat Res 80:122-130

4. Butts JJ, Katz R (1967) Theory of RBE for heavy ion bombardment of dry enzymes and viruses. Radiat Res 30:855-871

5. Kobetich EJ, Katz R (1969) Energy deposition by electron beams and $\delta$-rays. Phys Rev 170:257-265

6. Katz R, Ackerson B, Homayoonfar M, Scharma SC (1971) Inactivation of cells by heavy ion bombardment. Radiat Res 47:402-425

7. Cucinotta FA, Wilson JW, Shavers MR, Katz R (1996) Effects of track structure and cell inactivation on the calculation of heavy ion mutation rates in mammalian cells. Int J Radiat Biol 69:593-600

8. Katz R, Pinkerton FE (1975) Response of nuclear emulsions to ionizing radiation. Nucl Instrum Methods 130: 105-119

9. Goodhead DT, Nikjoo H (1990) Current status of ultrasoft $\mathrm{X}$-rays and track structure analysis as tools for testing and developing biophysical models of radiation action. Radiat Prot Dos 31:343-350

10. Nikjoo H, Goodhead DT, Charlton DE, Paretzke HG (1991) Energy deposition in small cylindrical targets by monenergetic electrons. Int J Radiat Biol 60:739-756

11. Frankenberg D, Kuhn H, Frakenberg-Schwanger M, Lenhard W, Beckonert S (1995) $0.3 \mathrm{keV}$ Carbon K ultrasoft X-rays are four times more effective than gamma-rays when inducing cell transformation at low doses. Int J Radiat Biol 68:593-601

12. Hamm RN, Turner JE, Wright HA, Ritchie RH (1979) Heavyion track structure in silicon. IEEE Trans Nuclear Sci 26:48924895

13. Cucinotta FA, Wilson JW, Shinn JL, Badavi FF, Badhwar GD (1996) Effects of target fragmenation on evaluation of LET spectrum from space radiation: implications for space radiation protection studies. Radiat Meas 26:923-934

14. Zeitlin C, Heilbronn L, Miller J (1998) Detailed characterization of the $1087 \mathrm{MeV} /$ nucleon iron-56 beam used for radiobiology at the alternating gradient synchrotron. Radiat Res 149:560569

15. Cucinotta FA, Katz R, Wilson JW, Dubey RR (1995) Radial dose distributions in the delta-ray theory of track structures. In: Gray
TJ, Starace AF (eds) Proceedings of two-center effects in ionatom collisions. AIP Conference Proceedings, pp 245-265

16. Rudd ME (1989) User friendly model for the electron distribution of electrons from proton or electron collisions. Nucl Tracks Radiat Meas 16:213-218

17. Kobetich EJ, Katz R (1969) Electron energy dissipation. Nucl Instrum Methods 71:226-230

18. Tabata T, Okabe S (1972) Generalized semiempirical equations for the extrapolated range of electrons. Nucl Instrum Methods 103: 85-91

19. Brandt W, Ritchie RH (1974) Primary processes in the physical stage. In: Cooper RD, Woods R (eds) Physical mechanisms in radiation biology. Tech Info Center, US Atomic Energy Commission Springfield, Virginia, pp 20-29

20. Bethe HA, Rose ME, Smith LP (1938) The multiple scattering of electrons. Proc Am Philos Soc 78:573-585

21. LaVerne JA Mozumder A (1983) Penetration of low-energy electrons in water. Radiat Res 96:219-234

22. Wingate CL, Baum JW (1976) Measured radial distributions of dose and LET for alpha and proton beams in hydrogen and tissue-equivalent gas. Radiat Res 65:1-19

23. Paretzke HG (1980) Advances in energy deposition theory. In: Thomas RH, Perez-Mendez V (eds) Advances in radiation protection and dosimetry in medicince. Plenum, New York, pp 51-73

24. Zaider M, Brenner DJ, Wilson WE (1983) The application of track calculations to radiobiology. I. Monte-Carlo simulation of proton tracks. Radiat Res 95:231-247

25. Krämer M, Kraft G (1994) Heavy ion track structure calculations. Radiat Environ Biophys 33:91-109

26. Varma MN, Baum JW (1980) Energy deposition in nanometer regions by $377 \mathrm{MeV} /$ nucleon $^{20} \mathrm{Ne}$ ions. Radiat Res $81: 355-363$

27. Belli M, Cera F, Cherubini R, Haque AMI, Ianzini F, Moschini G, Sapora O, Simone G, Tabocchini MA, Tiveron P (1993) Inactivation and mutation induction in V79 cells by low energy protons: re-evaluation of the results at the LNL facility. Int $\mathbf{J}$ Radiat Biol 63:331-337

28. Folkard M, Prise KM, Vojnovic B, Newman HC, Roper MJ, Michael BD (1996) Inactivation of V79 cells by low-energy protons, deuterons, and helium-3 ions. Int J Radiat Biol 69:729-738

29. Aufderheide E, Rink H, Hieber L, Kraft G (1987) Heavy ion effects on cellular DNA: strand break induction and repair in cultured diploid lens epithelial cells. Int J Radiat Biol 51:779-785

30. Kiefer J, Schneider E (1994) Mutation induction by heavy ions. Adv Space Res 14:257-265

31. Kellerer AM (1971) An assessment of the wall effects in microdosimetric measurements. Radiat Res 47:377-386

32. Dicello JF, Wasiloek M, Zaider M (1991) Measured microdosimetric spectra of energetic ion beams of $\mathrm{Fe}, \mathrm{Ar}, \mathrm{Ne}$, and $\mathrm{C}$ : limitation of LET distributions and quality factors in space research and radiation effects. IEEE Trans Nucl Sci 38: 1203-1209

33. Booz J (1976) Microdosimetric spectra and parameters of lowLET radiations. (CEC Report EUR-5452) Proc Symp Microdosimetry $5^{\text {th }}$, Verbania Pallanza, pp 311-314

34. Metting NF, et al (1988) Microdosimetry near the trajectory of high-energy heavy ions. Radiat Res 116:183-195

35. Metting NF (1986) MSc thesis, University of Washington, Seattle, WA 